\title{
Exchange biasing in ferromagnetic amorphous wires: A controllable micromagnetic configuration
}

\author{
J. J. Freijo, ${ }^{\text {a) }}$ A. Hernando, and M. Vázquez \\ Instituto de Magnetismo Aplicado, UCM-RENFE-CSIC P.O. Box 155, 28230 Las Rozas, Madrid, Spain
}

A. Méndez and V. R. Ramanan

ABB Electric Systems Technology Institute, 1021 Main Campus Drive, Raleigh, North Carolina 27606

(Received 26 August 1998; accepted for publication 4 January 1999)

\begin{abstract}
Nonmagnetostrictive Co-rich amorphous wires, $130 \mu \mathrm{m}$ in diameter, were stress annealed to induce a transverse anisotropy. The resultant structure consists of a circular-magnetization single domain except in a thin core, $2 \mu \mathrm{m}$ in diameter, where magnetization is axially oriented. The wall interconnecting the outer shell with the core magnetization induces a local helical anisotropy during the annealing. As a consequence, a strong link between the magnetization directions in both regions appears. It is shown that direct current axial fields produce remarkable shifts of the circular hysteresis loops. (C) 1999 American Institute of Physics. [S0003-6951(99)01109-2]
\end{abstract}

Amorphous ferromagnetic materials are characterized by the lack of structural order that gives rise to a negligible structural magnetic anisotropy. This characteristic, together with the high atomic mobility of the amorphous state, allows us to induce macroscopic anisotropies by means of suitable field and/or stress annealing treatments. ${ }^{1}$ This property becomes particularly interesting since the control of the anisotropy leads to the control of the magnetic behavior. Moreover, as reported in this letter, amorphous ferromagnets with negligible magnetostriction are ideal materials to tailor micromagnetic configurations with extremely interesting magnetization process characteristics from both fundamental and applications scopes.

Amorphous wires are currently obtained by the rotatingwater-quenching technique. ${ }^{2}$ The study of these materials has been a topic of considerable interest during the last decade as they present outstanding properties, such as magnetic bistability ${ }^{3}$ and giant magnetoimpedance, ${ }^{4}$ which make them particularly interesting for a number of sensing applications. A detailed review of the characteristics of these materials can be found in Ref. 5 .

This work presents a peculiar exchange bias effect observed on Co-rich amorphous wires exhibiting a transverse anisotropy, induced by stress annealing. This effect gives rise to a noticeable shift of the circular hysteresis loops when a direct current (dc) axial bias field is applied. Up to the present date, shifts in the hysteresis loops have been reported in (i) composite antiferro (AF)/ferro (FM) or spin glass (SG)/ ferro (FM) materials, in which the AF or SG phases acts as a pinning for the ferromagnetic phase, ${ }^{6}$ this has been the origin of the spin valves structures; (ii) hard-soft two phase magnetic materials in which both the exchange and dipolar interactions are responsible of the above mentioned effect; ${ }^{7}$ and (iii) materials subjected to a dc bias field parallel to the driving field, as is the typical case of flux-gate magnetometers based on the hysteresis shift produced by a static field. ${ }^{8}$

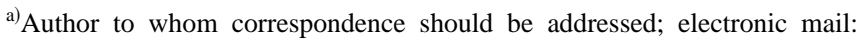
freijo@fenix.ima.csic.es
}

We have studied nearly zero magnetostriction $\left(\mathrm{Co}_{96} \mathrm{Fe}_{6}\right)_{72.5} \mathrm{Si}_{12.5} \mathrm{~B}_{15}$ alloy wires treated using tension annealing at $450{ }^{\circ} \mathrm{C}$ in order to induce magnetic anisotropy ${ }^{9}$ (the thermal treatment was carried out under a $400 \mathrm{MPa}$ tensile stress, value for which the transverse induced anisotropy overcomes any other residual anisotropy). The wires were kindly supplied by the Japanese company UNITIKA Inc. All the measurements were performed on wires $130 \mu \mathrm{m}$ in diameter and $30 \mathrm{~cm}$ in length.

The axial $\left(M_{z}-H_{z}\right)$ hysteresis loop was measured with a conventional induction technique hysteresis loop tracer at 80 $\mathrm{Hz}$. In order to obtain this loop, the magnetic field $\left(H_{z}\right)$ was applied along the wire axis using a long solenoid. The changes in the axial component of the magnetization $\left(M_{z}\right)$ were picked up with a coaxial 1000 turns secondary coil. In Fig. 1(a) the longitudinal hysteresis loop of the studied sample is displayed. The tension annealing process induces a transverse anisotropy as shown by the linear nonhysteretic loop, characteristic of coherent rotation when a magnetic field is applied along the hard axis direction. From the experimental anisotropy field which reaches a value close to $500 \mathrm{~A} \mathrm{~m}^{-1}$ and by considering the value of the spontaneous magnetization $M_{s}=5.6 \times 10^{5} \mathrm{Am}^{-1}$, the deduced anisotropy constant is $K=200 \mathrm{~J} \mathrm{~m}^{-3}$.

The circular magnetization versus circular magnetic field hysteresis curve $\left(M_{\phi}-H_{\phi}\right)$ was also measured and is displayed in Fig. 1(b). In this case, the circular magnetic field

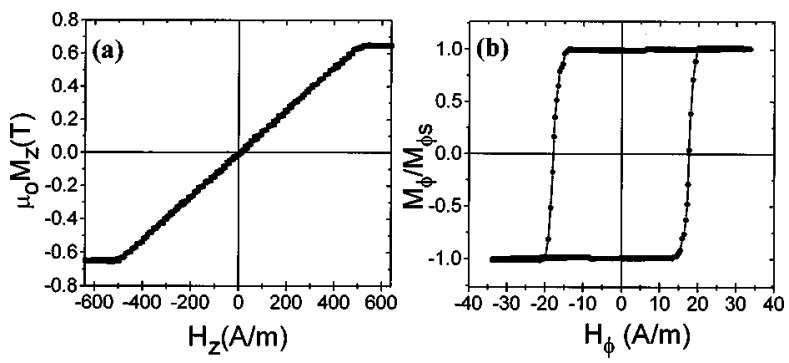

FIG. 1. (a) Axial $\left(M_{z}-H_{z}\right)$ and (b) circular $\left(M_{\phi}-H_{\phi}\right)$ hysteresis loops for a 30-cm-long sample annealed under $400 \mathrm{MPa}$ tensile stress. 
$\left(H_{\phi}\right)$ was created by driving a current through the wire. The changes in the circular component of the magnetization $\left(M_{\phi}\right)$ produce a voltage drop between the ends of the wire which was integrated and plotted versus the circular field. The measurement technique has been fully described elsewhere. ${ }^{10}$ The magnetization has been plotted as a function of the magnetic field at the surface of the wire. The square shape of the loop, typical of an easy axis magnetization process, is due to the circular anisotropy induced by the thermal treatment. The value of the circular coercive field $\left(H_{C \phi}\right)=18 \mathrm{~A} \mathrm{~m}^{-1}$ is 0.04 times the anisotropy field obtained from the axial loops, which suggests that the reversal of the circular magnetization is produced by a domain wall nucleation-propagation process. The value of the normalized remanence $\left(M_{r} / M_{s}\right)$ equals unity. From this observation, we can conclude the existence of only one outer shell domain along the entire length of the wire $(30 \mathrm{~cm})$ in which the magnetization lies circularly oriented. ${ }^{11}$

Let us consider the amorphous wire as an infinite cylinder divided in concentric shells. For symmetry reasons, the magnetization in each shell is postulated to be oriented around the axis of the wire forming an angle $\varphi$ with it (the radial component of the magnetization was assumed to be zero to minimize the magnetostatic energy). Under these conditions, the magnetic energy density of the wire is given by the sum of anisotropy and exchange energies. Minimization of the energy leads to the following Euler equation:

$$
\left(\frac{d^{2} \varphi}{d r^{2}}\right)+\frac{1}{r}\left(\frac{d \varphi}{d r}\right)+\left(\frac{K}{A}-\frac{1}{2 r^{2}}\right) \sin 2 \varphi=0
$$

$A$ being the exchange constant, typically $10^{-11} \mathrm{~J} \mathrm{~m}^{-1}, r$ the radial coordinate, and $K$ the transverse anisotropy constant. The first two terms account for the radial exchange, whereas the azimuthal exchange can be gathered as an effective anisotropy term with $k^{*}=\left(K-A / r^{2}\right)$. Equation (1), points out that, if we neglect the radial exchange, the easy magnetization direction is circular $(\varphi=\pi / 2$ or $3 \pi / 2)$ for $r$ $>(A / 2 K)^{1 / 2}$ and axial $(\varphi=0$ or $\pi)$ for $r<(A / 2 K)^{1 / 2}$, respectively. The physical meaning of these solutions can be understood as follows; for small $r$ if the magnetization orientation were circular, thus minimizing the anisotropy energy, the azimuthal exchange which varies as $1 / r^{2}$ should increase enough to reach an energetically unfavorable balance. The sharp $\pi / 2$ transition of the easy axes orientation at $r$ $=(A / 2 K)^{1 / 2}$ is smoothed by the radial exchange as illustrated in Fig. 2. Equation (1) has been solved using a finite difference method. ${ }^{12}$ Results for different values of the parameter $\xi=K / A$ are shown in Fig. 2. It is worthwhile noticing that for our sample with $K=200 \mathrm{~J} \mathrm{~m}^{-3}$, or $\xi=20 \mu \mathrm{m}^{-2}$, the inner core plus the wall extends approximately $1 \mu \mathrm{m}$ around the axis. Therefore, only in a volume fraction of around $0.03 \%$ the magnetization lies out of the circular direction. This negligible volume fraction accounts for the lack of remanence in the axial loop as well as for the squareness of the circular magnetization loops.

According to Eq. (1), in the transition zone the effective anisotropy vanishes, $k^{*}=0$ at $r=(A / 2 K)^{1 / 2}$. Therefore, the reversal of the circular magnetization is expected to start in this zone and then to propagate toward the outer surface.

The experimental $\left(M_{\phi}-H_{z}\right)$ hysteresis curve (Fig. 3)

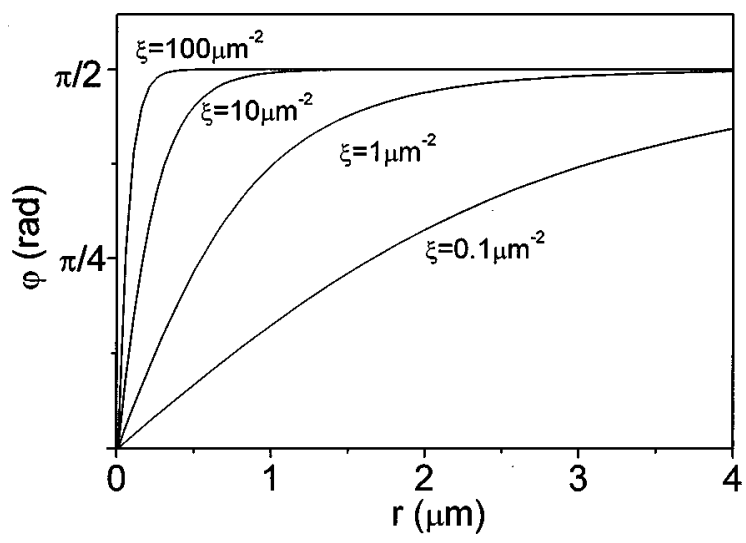

FIG. 2. Angle $(\varphi)$ between the magnetization and the wire axis as a function of the radial coordinate as obtained after solving Eq. (1). The parameter $\xi$ $=K / A$ represents the anisotropy-exchange ratio.

was obtained by applying a magnetic field parallel to the axis of the wire with a long solenoid and measuring the change in the circular component of the magnetization as explained in the case of the circular loop hysteresis loop. This curve provides a deeper insight of the magnetization process. In particular, it shows the existence of a preferred helical distribution in the wall region. From the remanent state, the axial magnetization process is due to reversible rotation of the circularly oriented outer shell towards the axial direction (see inset Fig. 3). However, for a critical value of the field, $H_{\text {cr }}$ $=500 \mathrm{~A} \mathrm{~m}^{-1}$, the circular magnetization is reversed and an effective hysteresis appears. The reversal of the circular magnetization is originated by the action of an axial field that can be interpreted as illustrated in Fig. 4(a). In Fig. 4 the two cases are shown: (a) When the wall has induced a localized helical anisotropy during the annealing, ${ }^{13}$ the magnetic moment of the wall at $r$ can easily lie only along $\varphi$ or $\varphi+\pi$ and, thus, the reversal of axial magnetization tends to reverse the circular magnetization. The same argument leads to conclude that the reversal of the circular magnetization induces the reversal of the axial one. (b) However, when the localized helical anisotropy is negligible the magnetic moments located at the wall can orientate with the same energy along any $\varphi$ as $-\varphi$ or $\pi-\varphi$ and the reversal of the circular and

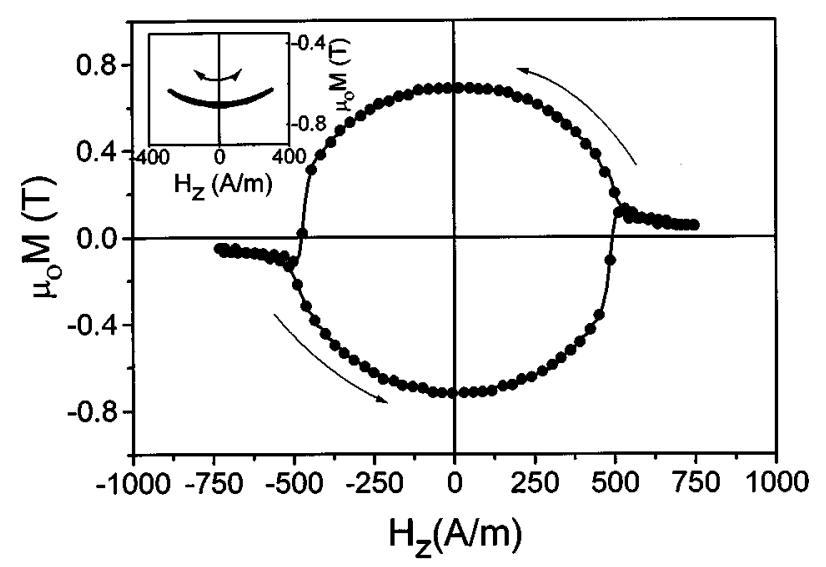

FIG. 3. Circular magnetization vs axial field hysteresis loop $\left(M_{\phi}-H_{z}\right)$ for a 30-cm-long sample annealed under $400 \mathrm{MPa}$ stress. Inset: The minor $\left(M_{\phi}-H_{z}\right)$ loop points out the reversible character of the magnetization process until $H_{\text {cr }}$ is reached.

Downloaded 09 Mar 2010 to 161.111 .180 .191 . Redistribution subject to AIP license or copyright; see http://apl.aip.org/apl/copyright.jsp 

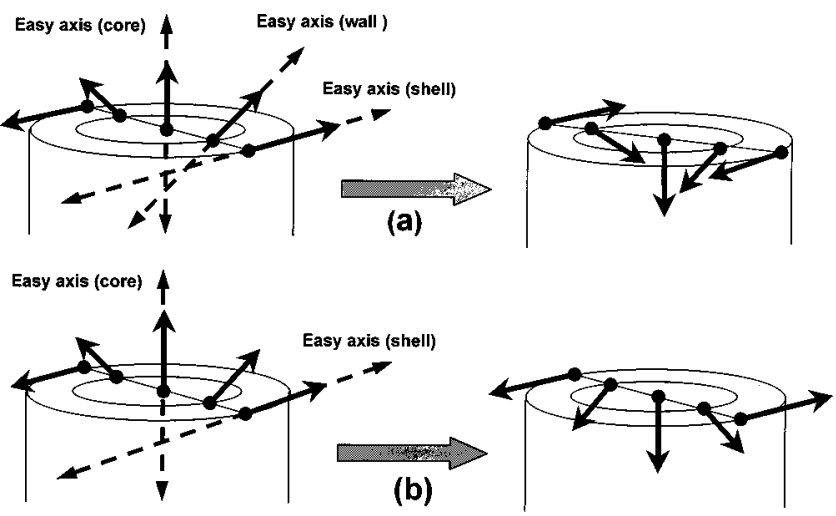

FIG. 4. Reversal of the core magnetization when an axial field is applied: (a) If an induced anisotropy is present at the wall region the inversion of the core causes the inversion of the shell; (b) if such anisotropy is not present the core reversal occurs independently. The dotted lines represent the easy axes at the different regions.

axial magnetization components are carried out independently.

The $\left(M_{\phi}-H_{z}\right)$ loop has shown the existence of a correlation between the axial and circular components of the wire. The origin of such correlation is the preferred helical path induced at the wall during the annealing and frozen in after cooling. ${ }^{14}$

A consequence of the preferred helical path of the wall is that deviations of the symmetry in both axial and circular hysteresis loops are expected when applying circular and axial dc bias field, respectively. The effect of an axial dc field on the circular magnetization process can be seen in Fig. 5. When applying an axial field lower than the anisotropy field, the magnetization is tilted out of the circular di-

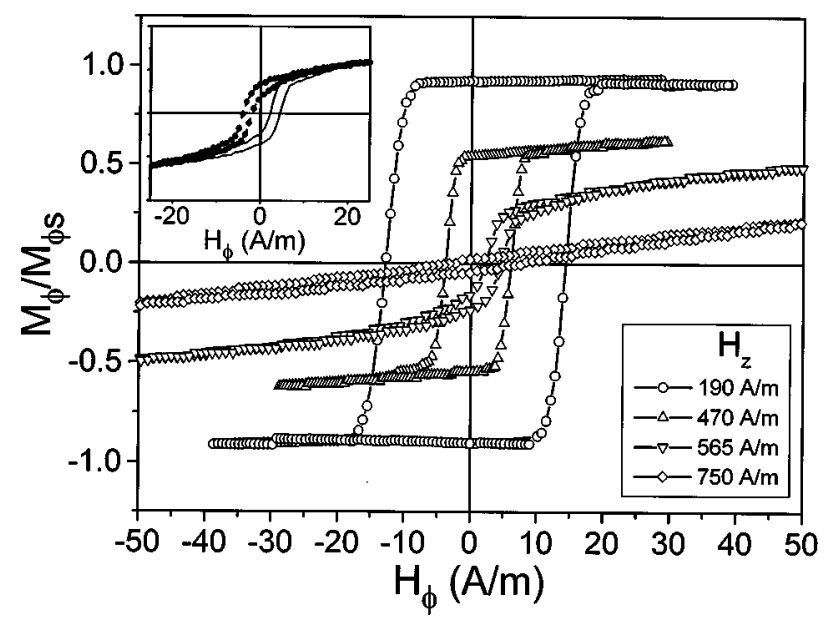

FIG. 5. Circular $\left(M_{\phi}-H_{\phi}\right)$ hysteresis loops for different biasing axial fields. Inset: two circular hysteresis loops corresponding to applied biasing fields of the same magnitude but opposite axial directions. It can be observed that the direction of the shift is correlated with the direction of the biasing field, as expected from the existence of a helical anisotropy. rection so decreasing the remanence and, besides the irreversible switching process, an additional rotation of the magnetization back to the easy direction is necessary to reach saturation. This is clearly deduced from the loop corresponding to $H_{z}=470 \mathrm{~A} \mathrm{~m}^{-1}$ that approaches to saturation following a reversible rotation process.

Nevertheless, the most remarkable effect is that the dc axial field introduces a noticeable shift in the circular hysteresis loops along the horizontal direction, as is clearly observed for $H_{z}=565 \mathrm{~A} \mathrm{~m}^{-1}$. The shift is towards the opposite direction when the dc field direction is reversed (see inset in Fig. 5). This shift points out the exchange bias effect of the wire core axial magnetization on the outer shell circular magnetization. When the axial field defines the axial magnetization direction it is also defining a preferred circular direction through the coupling carried by the anisotropic wall. For further increasing bias dc fields, the longitudinal saturation is reached and the effect of the circular field is restricted to induce a reversible rotation toward the circular direction, as illustrated in the hysteresis loop corresponding to $H_{z}$ $=750 \mathrm{~A} \mathrm{~m}^{-1}$.

In summary, it has been shown that under suitable stress annealing it is possible to create a circular single domain structure in amorphous wires. A thin wire core exhibits axial magnetization direction. Radial exchange induces the formation of a $90^{\circ}$ wall separating both regions. The anisotropy induced by the wall itself gives rise to a strong correlation between the direction of both magnetization components.

${ }^{1}$ B. W. Corb, R. C. O’Handley, J. Megusar, and N. J. Grant, Phys. Rev. Lett. 51, 1386 (1983); O. V. Nielsen, IEEE Trans. Magn. MAG-21, 2008 (1985); J. M. Riveiro, V. Madurga, and A. Hernando, Phys. Rev. B 39, 11950 (1989); as a review see also A. Hernando and M. Vázquez in H. H. Liebermann Rapidly Solidified Alloys (Marcel Dekker, New York, 1993), pp. 553.

${ }^{2}$ I. Ogasawara and S. Ueno, IEEE Trans. Magn. 31, 1219 (1995).

${ }^{3}$ K. Mohri, F. B. Humphrey, J. Yamasaki, and K. Okamura, IEEE Trans. Magn. MAG-20, 1409 (1984); M. Vázquez and D. X. Chen, ibid. 31, 1229 (1995).

${ }^{4}$ R. S. Beach and A. E. Berkowitz, Appl. Phys. Lett. 64, 3652 (1994); J. Velázquez, M. Vázquez, D. X. Chen, and A. Hernando, Phys. Rev. B 50, 16737 (1994).

${ }^{5}$ M. Vázquez and A. Hernando, J. Phys. D 29, 939 (1996).

${ }^{6}$ W. H. Meiklejohn and C. P. Bean, Phys. Rev. 105, 904 (1957); R. H. Kodama, S. A. Makhlouf, and A. E. Bekowitz, Phys. Rev. Lett. 79, 1393 (1997).

${ }^{7}$ D. X. Chen and X. Pan, Acta Metall. Sin. 19, 176 (1983)

${ }^{8}$ F. Primdall, IEEE Trans. Magn. MAG-6, 376 (1970).

${ }^{9}$ H. Theuss, B. Hofmann, C. Gomez-Polo, M. Vázquez, and H. Kronmüller, J. Magn. Magn. Mater. 145, 165 (1995).

${ }^{10}$ A. Hernando and J. M. Barandiarán, J. Phys. D 11, 1539 (1978).

${ }^{11}$ The single domain character of the outer shell is noticeable since bamboolike domain structures have been observed in as cast nearly zero or negative magnetostrictive wires with transverse easy axis.

${ }^{12}$ L. Collatz, The Numerical Treatment of Differential Equations (Springer, Berlin, 1966), p. 141.

${ }^{13}$ C. Aroca, P. Sánchez, and E. López, Phys. Rev. B 34, 490 (1986); M. Maicas, E. López, P. Sanchez, M. C. Sánchez, and C. Aroca, ibid. 47, 3180 (1993).

${ }^{14}$ M. Liniers, V. Madurga, M. Vázquez, and A. Hernando, Phys. Rev. B 31, 4425 (1985). 